Open Access

\title{
Effects of a grape-supplemented diet on proliferation and Wnt signaling in the colonic mucosa are greatest for those over age 50 and with high arginine consumption

Randall F. Holcombe ${ }^{1 *}$, Micaela Martinez ${ }^{2}$, Kestutis Planutis ${ }^{1}$ and Marina Planutiene ${ }^{1}$

\begin{abstract}
A diet rich in fruits and vegetables, and a grape-derived compound, resveratrol, have been linked to a reduced incidence of colon cancer. In vitro and in vivo, resveratrol suppresses Wht signaling, a pathway constitutively activated in over $85 \%$ of colon cancers.

Thirty participants were placed on a low resveratrol diet and subsequently allocated to one of three groups ingesting 1/3-to-1 lb (0.15-0.45 kg) of grapes per day for 2 weeks. Dietary information was collected via 24-h recall. Colon biopsies for biomarker analysis were obtained pre- and post-grape and evaluated for the expression of Wnt pathway target genes and for markers of proliferation by RT-PCR and immunohistochemistry.

Participants lost an average of $2.6 \mathrm{lb}(1.2 \mathrm{~kg}, p=0.0018)$ during the period of grape ingestion. The expression of CyclinD1 $(p<0 \cdot 01), A X I N 2, C D 133(p=0.02)$ and Ki67 $(p=0.002)$ were all reduced after grape ingestion.

Individuals over 50 years of age and those with high dietary arginine consumption had increased basal expression of CyclinD1, AXIN2, cMYC and CD133 ( $p$ value range 0.04 to $<0.001$ ) that, following grape ingestion, were reduced to levels seen in younger participants.

The reduction in Wht signaling and mucosal proliferation seen following short-term ingestion of $1 / 3-1 \mathrm{lb}(0.15-0.45 \mathrm{~kg})$ of grapes per day may reduce the risk of mutational events that can facilitate colon carcinogenesis. The potential benefit is most marked for high-risk older individuals and individuals whose diet is high in arginine intake. Dietary grape supplementation may play a role in colon cancer prevention for high-risk individuals.
\end{abstract}

Keywords: Diet, Cancer prevention, Resveratrol, Colon cancer, Aging, Arginine

\section{Introduction}

Studies suggest strongly that a diet rich in fruits and vegetables leads to a lower risk of colorectal cancer (CRC) $[1,2]$. Grape seeds and other grape-based products have purported CRC prevention activity [3], are rich in polyphenols and contain resveratrol, anthocyanins, catechins, quercetin and numerous other compounds with chemopreventive potential [4]. The most intensively studied component in grapes is resveratrol which suppresses PI3-kinase, AKT and NF-kB signaling pathways [5]

\footnotetext{
* Correspondence: Randall.holcombe@mssm.edu

'Division of Hematology \& Medical Oncology, Tisch Cancer Institute, Icahn School of Medicine at Mount Sinai, One Gustav L. Levy Place, Box 1128, New York, NY 10029, USA

Full list of author information is available at the end of the article
}

and may affect tumor growth by a myriad of other mechanisms as well $[6,7]$. Systemic administration of resveratrol has been shown to inhibit the growth of intestinal tumors in several different rodent cancer models $[8,9]$. For colon cancer prevention, effects are seen over a wide variety of dose ranges depending on individual studies. Tessotore [10] demonstrated activity of very low dose resveratrol of $0.2 \mathrm{mg} / \mathrm{kg} /$ day in reducing aberrant crypt foci (ACF) in the colon in an azoymethane-induced tumor model. In another carcinogen-based model, utilizing 1,2-dimethylhydrazine, resveratrol at $8 \mathrm{mg} / \mathrm{kg} /$ day reduced both ACF and colonic tumors [11] as did gavage of $60 \mathrm{mg} / \mathrm{kg}$ body weight [12]. In genetic models utilizing the APCmin/+ mouse, which harbors a single allele mutation in apc and therefore has intrinsically activated Wnt signaling, 
Schneider [13] demonstrated profound activity at dosages as low as $0.3 \mathrm{mg} / \mathrm{mouse} /$ day in reducing intestinal tumors. In this study, expression of Wnt target gene cyclinD1 as well as other markers of cell cycling was reduced.

Resveratrol, even at low concentrations, blocks Wnt signaling in colon cancer cells in vitro [14]. This pathway is activated in over $85 \%$ of CRC making it an attractive target for a colon cancer prevention agent. Resveratrolrich freeze-dried grape powder has been utilized in a pilot study in normal human volunteers and was found to down-regulate the expression of Wnt pathway target genes CyclinD1 and AXIN2 in colonic mucosa [15]. However, low bioavailability of individual compounds such as resveratrol often results in systemic concentrations too low to be clinically active [16]. Therefore, it is important in consideration of dietary approaches to cancer prevention to consider the aggregate activity of all of the bioactive components in a particular foodstuff [17] and not just single purified compounds.

This phase I study was undertaken to evaluate the potential role of a grape-supplemented diet for CRC prevention. The endpoints were biologic biomarkers of proliferation and Wnt signaling in colonic mucosa. During the study, detailed dietary information was collected and analyzed. This study is unique in that the effects of the complete foodstuff, rather than a refined component (ie grape seed extract) or individual substance (ie resveratrol) on biologically relevant cancer prevention endpoints is being investigated.

\section{Materials and methods}

\section{Clinical trial design and conduct}

30 healthy non-diabetic volunteers were enrolled over a period of 9 months for this study (clinical trial NCT00578396) which was approved by the University of California, Irvine institutional review board (ethics committee). Written informed consent was obtained for all participants. One potential subject was excluded from the study because of an elevated blood sugar discovered during eligibility testing that included a complete blood count and chemistry profile. This individual was referred for evaluation of occult diabetes. All participants had an initial consultation with a dietitian and were placed onto a low resveratrol diet (no grapes, wine, raisins, peanuts, peanut butter or cranberries) for 4 weeks. The controlled resveratrol diet was instituted as a base diet prior to sample acquisition and continued during the phase of grape ingestion. This was done to ensure minimal impact of potential dietary changes during the interventional component of the study. $24 \mathrm{~h}$ dietary recall evaluations were performed 3 times during days 1-14, and three more times during days $15-30$, with data entered into a dietary software program "Nutrition Data System for Research (NDSR) Versions 2006-2008" produced by the University of Minnesota. During days 15-30, participants were assigned to one of three grape consumption cohorts: $1 / 3 \mathrm{lb}, 2 / 3 \mathrm{lb}$ or $1 \mathrm{lb}(0.15 \mathrm{~kg}$, $0.30 \mathrm{~kg}, 0.45 \mathrm{~kg}$ ) of grapes to be ingested each day. These amounts were selected by the investigators because $1 / 3 \mathrm{lb}$ of grapes is equivalent to one "serving" as defined by the United States Department of Agriculture (USDA). Each patient was given a voucher redeemable at a local grocery store for red, seedless grapes and a digital kitchen scale. Compliance with grape consumption was ascertained at the time of the $24 \mathrm{~h}$ recall evaluations and again at the end of subject participation. Assignment into the cohorts was determined by a random sequential enrollment algorithm. Limited flexible sigmoidoscopies were performed on day 15 ("pre-grape") and on day 30 ("post-grape"). Two-to-four rectal mucosal biopsies were obtained for RNA and immunohistochemical analysis. See Fig. 1 for a schematic of the clinical trial design.

\section{4 hour dietary recall}

Nutrition data system for research (NDSR) is a dietary analysis program designed for the collection and analyses of 24-h dietary recalls and the analysis of food records, menus, and recipes. Nutrient intake from both food and supplemental sources are captured and quantified. Dietary interviews took place over the telephone and/or in person and data was entered directly into NDSR. Food portion estimation visual aids and a kitchen scale were provided to respondents to assist in portion size estimation.

\section{Quantitative Real-time PCR (qRT-PCR)}

RNA was extracted from one-to-two of the biopsies obtained from each of the "pre-grape" and "post-grape" sigmoidoscopies using standard methodologies. Rectal mucosal biopsies were analyzed by quantitative real-time PCR for the expression of genes associated with proliferation and Wnt signaling including: ornithine decarboxylase (ODC1), nemo-like kinase (NLK), cJUN, cMYC, cyclinD1 (CCND1), lymphoid enhancing factor-1 (LEF1, TCF7L3), axinII (AXIN2), forkhead box protein 3 (FOXO3) and CD133 (Prominin1, PROM1). Primer pairs were obtained from Qiagen (Valencia, CA) with cycling parameters as defined by the manufacturer. Wnt target gene mRNA levels were all normalized to the housekeeping gene ribosomal RNA (rRNA). The relative RNA expression was calculated by the comparative threshold cycle method. All experiments were repeated in triplicate.

\section{Immunohistochemical analysis}

One-to-two of the biopsies obtained from each of the "pre-grape" and "post-grape" sigmoidoscopies were immediately placed into $10 \%$ formalin for processing and 


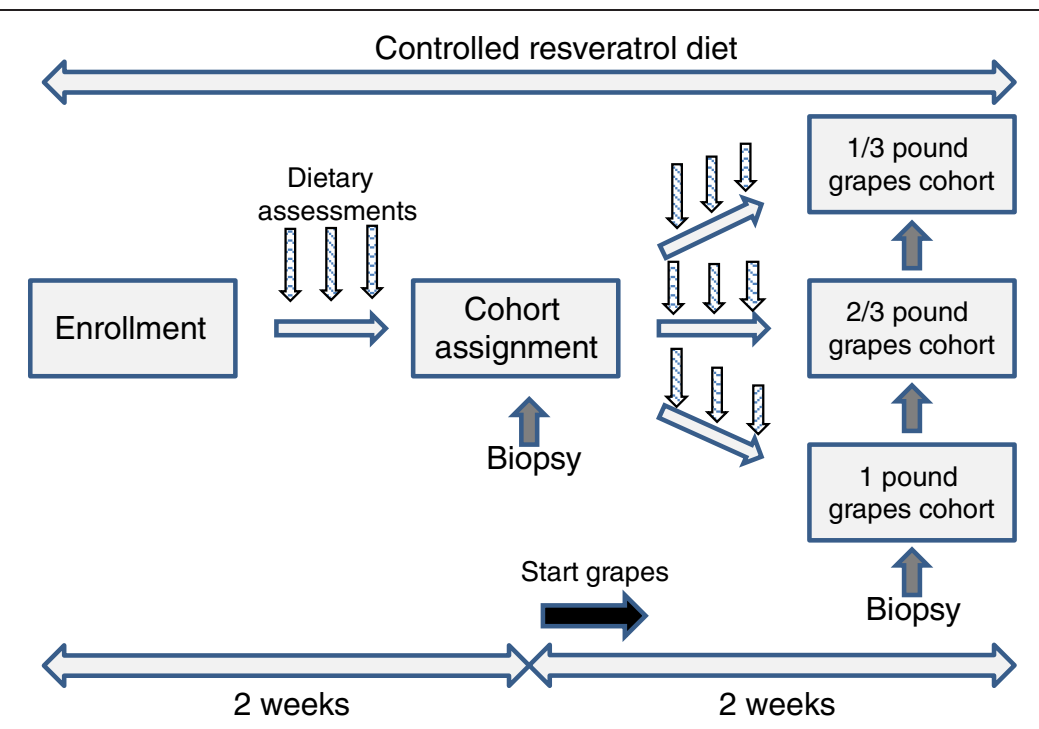

Fig. 1 Schematic of the clinical trial design. The controlled reservatrol diet was maintained throughout the 4 week period. Grape ingestion occurred during the final 2 weeks

subsequently paraffin embedded, sectioned, and placed onto slides for Ki67 immunohistochemistry (IHC). The percentage of cells expressing Ki67 in the lower 1/3 of colonic crypts was determined by fluorescence confocal microscopy. A minimum of 50 crypts were evaluated for each set of biopsies.

\section{Statistical analysis}

Patient characteristics were compared with 1-way ANOVA with a Newman-Keuls post-test. Dietary changes seen with grape supplementation, pre- and post-grape gene expression by qRT-PCR and Ki67 expression scored by IHC were analyzed with a Wilcoxon matched pairs

Table 1 Participant characteristics

\begin{tabular}{|c|c|c|c|c|c|}
\hline & $\begin{array}{l}\text { Overall group } \\
\text { (mean } \pm \text { SE) }\end{array}$ & $\begin{array}{l}1 \text { pound cohort } \\
\text { (mean } \pm \mathrm{SE} \text { ) }\end{array}$ & $\begin{array}{l}2 \text { pound cohort } \\
\text { (mean } \pm \mathrm{SE} \text { ) }\end{array}$ & $\begin{array}{l}3 \text { pound cohort } \\
\text { (mean } \pm \mathrm{SE} \text { ) }\end{array}$ & Significance $(p)^{*}$ \\
\hline Age & $43 \cdot 28 \pm 2 \cdot 28$ & $46 \cdot 00 \pm 3 \cdot 02$ & $43 \cdot 45 \pm 4 \cdot 79$ & $40 \cdot 10 \pm 3 \cdot 98$ & NS \\
\hline Initial weight (lb) & $172 \cdot 1 \pm 6 \cdot 55$ & $175 \cdot 3 \pm 16 \cdot 30$ & $171 \cdot 4 \pm 8 \cdot 22$ & $169 \cdot 2 \pm 7 \cdot 67$ & NS \\
\hline BMI & $28 \cdot 12 \pm 1 \cdot 07$ & $27 \cdot 89 \pm 2 \cdot 42$ & $27 \cdot 58 \pm 1 \cdot 63$ & $29 \cdot 01 \pm 1 \cdot 09$ & NS \\
\hline \multicolumn{6}{|l|}{ Gender (\#) } \\
\hline Male & 9 & 6 & 2 & 1 & \\
\hline Female & 21 & 4 & 8 & 9 & \\
\hline Total & 30 & 10 & 10 & 10 & \\
\hline \multicolumn{6}{|l|}{ Race/Ethnicity (\#) } \\
\hline White & 18 & 5 & 7 & 6 & \\
\hline Black & 2 & 1 & 1 & 0 & \\
\hline Chinese & 2 & 2 & 0 & 0 & \\
\hline Filipino & 1 & 1 & 0 & 0 & \\
\hline Hispanic & 4 & 0 & 0 & 4 & \\
\hline Japanese & 1 & 0 & 1 & 0 & \\
\hline Korean & 1 & 0 & 1 & 0 & \\
\hline Persian & 1 & 1 & 0 & 0 & \\
\hline Total & 30 & 10 & 10 & 10 & \\
\hline
\end{tabular}


signed-rank test. Linear regression analysis was utilized to generate $r^{2}$ and $p$ values for the comparison of AXIN2 and CCND1 expression. Comparison of expression levels stratified for age or for dietary arginine intake was undertaken with an unpaired $t$-test. Statistical significance was defined at a level of $\mathrm{p}<0 \cdot 05$.

\section{Results}

\section{Clinical trial and dietary analysis results}

There were no statistically significant differences between the any of the three cohorts of grape consumption for age, initial weight or body mass index (BMI; Table 1). Results related to dietary changes were not statistically different across the three cohorts and therefore data were combined for presentation in this report. More females participated in the study. The race/ethnicity of the subjects is depicted in Table 1 . No toxicities were reported for any of the 30 participants in the clinical trial. Toxicities were monitored by detailed interviews by research personnel. Compliance with the assigned grape consumption daily ingestion was $100 \%$ for the two week period being monitored. All participants said they were compliant with the dietary restrictions to limit resveratrolcontaining foods for the entire 4 week study period.

During the two weeks of grape consumption, participants lost an average of $2.6 \mathrm{lbs}(1.2 \mathrm{Kg} ; p=0.0018$; Fig. 2) despite significant increases in total carbohydrates, percent of calories from carbohydrates and total sugars

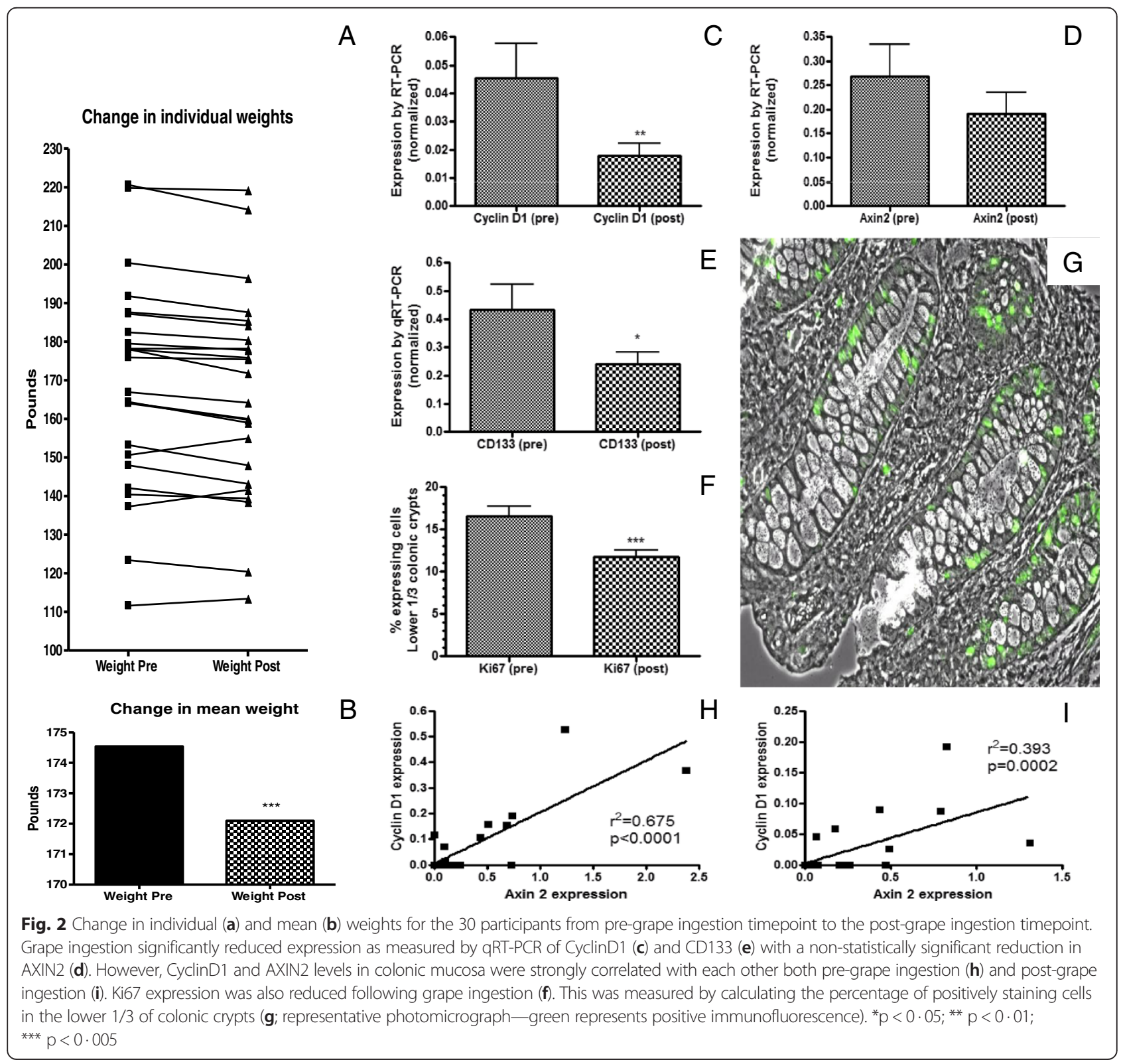


(Table 2). Overall KCALS were unchanged. Participants reported that they often felt "full", particularly those consuming $1 \mathrm{lb}(0.45 \mathrm{~kg})$ of grapes per day, and subjectively reported that they snacked less because of this. Interestingly, there was a significant reduction in the percentage of calories from fat $(35 \cdot 32$ to $30.77 \%)$ while ingesting grapes $(p=0 \cdot 011$; Table 2). Other significant changes included an increase in copper, potassium and vitamin B6 and a decrease in Biochanin A ingestion.

\section{Changes in markers of proliferation and Wnt signaling}

Results related to changes in proliferation and Wnt signaling markers were not statistically different across the three cohorts. In general the magnitude of the changes was slightly greater in the $1 \mathrm{lb} /$ day $(0.45 \mathrm{~kg})$ cohort than the $2 / 3 \mathrm{lb} /$ day $(0.30 \mathrm{~kg})$ and $1 / 3 \mathrm{lb} /$ day $(0.15 \mathrm{~kg})$ cohorts but the direction of the changes were consistent throughout all 3 groups. Each cohort contained only ten participants and therefore data for all participants were combined for presentation in this report. RT-PCR analysis revealed a significant reduction in the expression of CyclinD1, a proliferation marker and target gene for Wnt signaling, following grape ingestion (Fig. 2; p $<0.01$ ). A trend toward reduction in another Wnt target gene, AXIN2 was seen and the pre- and post-grape expression levels of CyclinD1 correlated significantly with the corresponding levels of AXIN2 (Fig. 1). The expression of CD133, a colonic stem cell marker was also significantly reduced $(p=0 \cdot 02)$. The expression of Ki67 protein, as measured by the percentage of expressing cells in the lower $1 / 3$ of colonic crypts, was significantly reduced following grape ingestion $(p=0 \cdot 002$; Fig. 2$)$. While the expression of several of the other genes tested had consistent trends, none, including cMYC, reached statistical significance.

\section{Effect of age and grape diet on markers of proliferation and Wnt signaling}

Data across all cohorts was stratified by age of participant. Twenty one participants were aged 18-49 and 9 were aged 50 and over. Several markers of Wnt signaling and proliferation were very significantly increased in older participants including CyclinD1 $(p=0 \cdot 022)$, AXIN2 $(p=0 \cdot 040)$ and $\mathrm{cMYC}(p=0 \cdot 006)$. The stem cell marker CD133 also had significantly increased expression in older individuals ( $p<0.001$; Fig. 3). As can be seen in Fig. 2, virtually all of the effect of grape ingestion was on the older participants, with no change in expression seen for any of these four markers in the 18-49 year old group and significant reductions seen in the 50 and older group. In most cases, the effect of grape ingestion was to reduce the levels of expression of these markers to a level seen basally in the younger participants (difference between "pre-younger" and "post-older" not significant in all cases).

\section{Effect of arginine intake and grape diet on markers of proliferation and Wnt signaling}

Participants were also stratified according to baseline arginine intake. The mean daily arginine intake was 4 . $5 \mathrm{gm} /$ day. Twenty one participants had arginine ingestion below the mean and nine participants had arginine ingestion above the mean. Expression of CyclinD1,

Table 2 Dietary changes observed with grape supplementation

\begin{tabular}{|c|c|c|c|c|}
\hline Parameter & Pre-grape baseline & At conclusion of grape intervention & Direction of change & Significance $(p)^{*}$ \\
\hline Weight (lbs) & $172 \cdot 1 \pm 6 \cdot 55$ & $170 \cdot 7 \pm 7 \cdot 65$ & $\downarrow$ & $0 \cdot 0018$ \\
\hline KCALS & $1960 \pm 131 \cdot 2$ & $1984 \pm 125 \cdot 1$ & $\leftrightarrow$ & NS \\
\hline Carbohydrate (gm) & $233 \cdot 4 \pm 15 \cdot 8$ & $271 \cdot 3 \pm 18 \cdot 2$ & $\uparrow$ & $0 \cdot 0048$ \\
\hline Calories from carbohydrate (\%) & $46 \cdot 99 \pm 1 \cdot 58$ & $51 \cdot 87 \pm 1 \cdot 53$ & $\uparrow$ & $0 \cdot 0089$ \\
\hline Total sugars (gm) & $92 \cdot 49 \pm 7 \cdot 86$ & $131 \cdot 9 \pm 9 \cdot 44$ & $\uparrow$ & $0 \cdot 0001$ \\
\hline Calories from fat (\%) & $35 \cdot 32 \pm 1 \cdot 44$ & $30 \cdot 77 \pm 1 \cdot 20$ & $\downarrow$ & 0.011 \\
\hline Copper (mg) & $1 \cdot 32 \pm 0 \cdot 13$ & $1 \cdot 53 \pm 0 \cdot 11$ & $\uparrow$ & 0.0035 \\
\hline Potassium (mg) & $2566 \pm 189$ & $3004 \pm 203$ & $\uparrow$ & $0 \cdot 0014$ \\
\hline Vitamin B6 (mg) & $1 \cdot 957 \pm 0 \cdot 16$ & $2 \cdot 254 \pm 0 \cdot 21$ & $\uparrow$ & 0.0193 \\
\hline Biochanin A (mg) & $0 \cdot 133 \pm 0 \cdot 07$ & $0.013 \pm 0 \cdot 01$ & $\downarrow$ & 0.0067 \\
\hline Folate (mcg) & $486 \cdot 2 \pm 41 \cdot 8$ & $433 \cdot 0 \pm 41 \cdot 1$ & $\leftrightarrow$ & NS \\
\hline Cholesterol (mg) & $289 \cdot 3 \pm 34 \cdot 93$ & $262 \cdot 0 \pm 28 \cdot 06$ & $\leftrightarrow$ & NS \\
\hline Genistein (mg) & $2 \cdot 53 \pm 1 \cdot 11$ & $0 \cdot 61 \pm 0 \cdot 20$ & $\leftrightarrow$ & NS \\
\hline Lycopene (mcg) & $4798 \pm 726$ & $4064 \pm 702$ & $\leftrightarrow$ & NS \\
\hline Arginine (gm) & $4 \cdot 503 \pm 0 \cdot 41$ & $4 \cdot 276 \pm 0 \cdot 34$ & $\leftrightarrow$ & NS \\
\hline
\end{tabular}



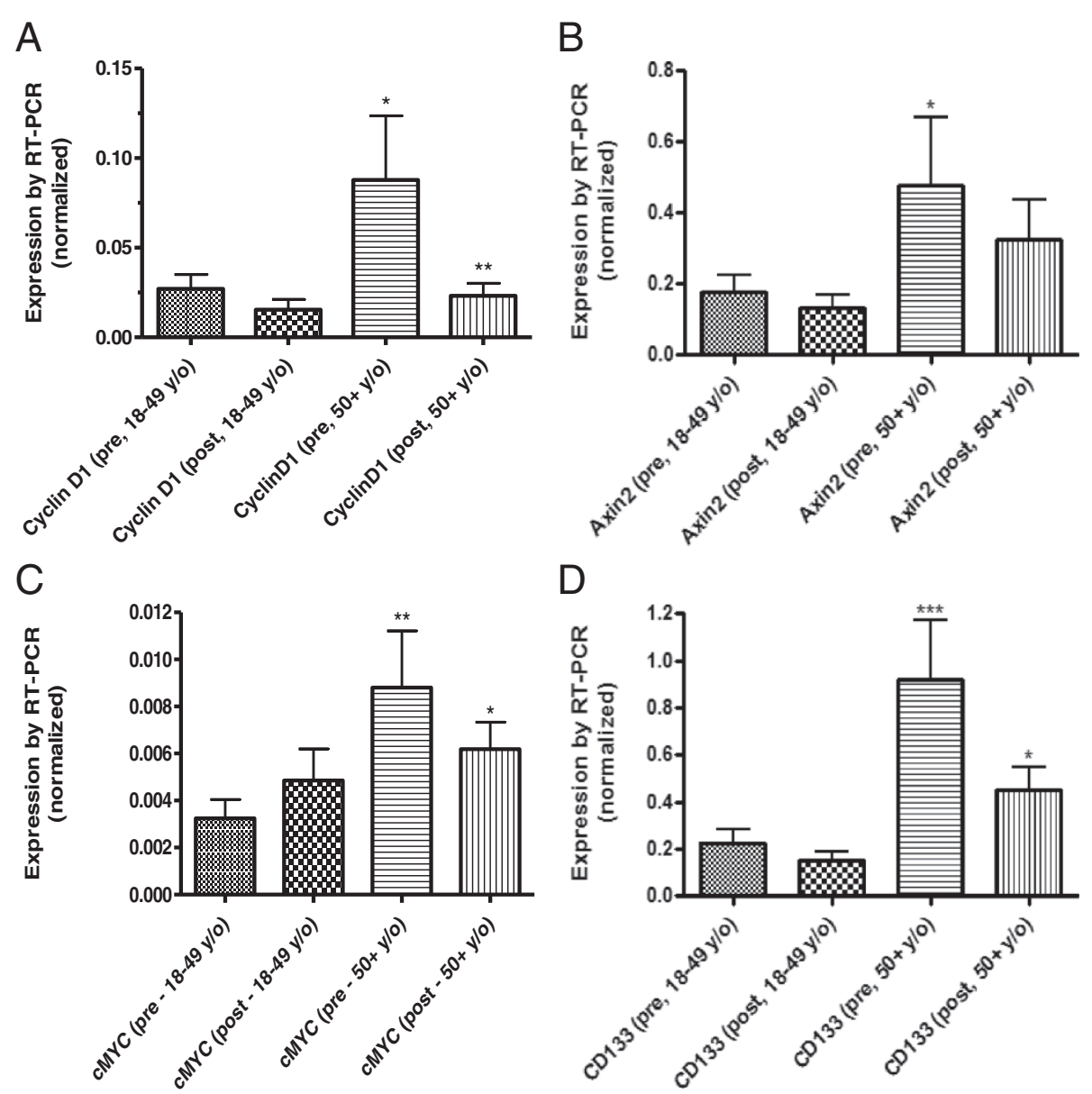

Fig. 3 Differences in expression of CyclinD1 (a), AXIN2 (b), CMYC (c) and CD133 (d) in participants below age 50 and 50 years old and above. Prior to grape ingestion, older individuals had significantly higher levels of CyclinD1, AXIN2, CMYC and CD133 than individuals aged 18-49. Grape ingestion in participants aged 50 and above led to a reduction in the expression CyclinD1 $(p<0 \cdot 01)$, AXIN2, CMYC and CD133. Levels of CyclinD1 and AXIN2 in participants aged 50 and above were reduced to levels seen prior to grape ingestion in 18-49 year olds. While reduced after grape ingestion, the level of expression of CMYC and CD133 in participants aged 50 and above remained significantly elevated when compared to the pre-grape ingestion levels of $18-49$ year olds. ${ }^{*} p<0 \cdot 05 ;{ }^{* *} p<0 \cdot 01 ;{ }^{* *} p<0 \cdot 001$

AXIN2, cMYC and CD133 were all significantly higher in patients with high baseline arginine intake compared to those with below average arginine intake ( $\mathrm{p}$ values ranging from $0 \cdot 04$ to $0 \cdot 002$; Fig. 4). Grape ingestion resulted in a significant reduction in CyclinDlexpression in volunteers with high baseline dietary arginine consumption $(\mathrm{P}<0.0039)$, achieving expression levels below the baseline CyclinD1 levels for patients in the low arginine group. Reductions following grape ingestion were also seen with cMYC, Axin2 and CD133 but these were not statistically significant.

\section{Discussion}

Participants were able to tolerate up to a pound of grapes per day for 2 weeks with full compliance and without any adverse consequences. No patients with diabetes were enrolled on the trial based on a recommendation from the
Institutional Review Board (Ethics Committee) because of the high sugar content of grapes. While on study, participants ingested less fat and lost, on average, $1.2 \mathrm{~kg}$ of weight. While weight loss with grapes has been reported previously in the popular press [18], to our knowledge this is the first confirmation of this effect under controlled clinical trial conditions.

The effects of a grape supplemented diet on Wnt signaling and markers of proliferation were consistent with prior in vitro [14] studies using resveratrol and an in vivo [15] study using resveratrol supplements and freeze-dried grape powder. Inhibition of Wnt signaling and reduced colonic mucosal proliferation suggests potential cancer preventative effects of a grape-containing diet. Whether these effects are due to resveratrol alone or other constituents of grapes, or a combination of constituents, remains to be defined. This study was of short 


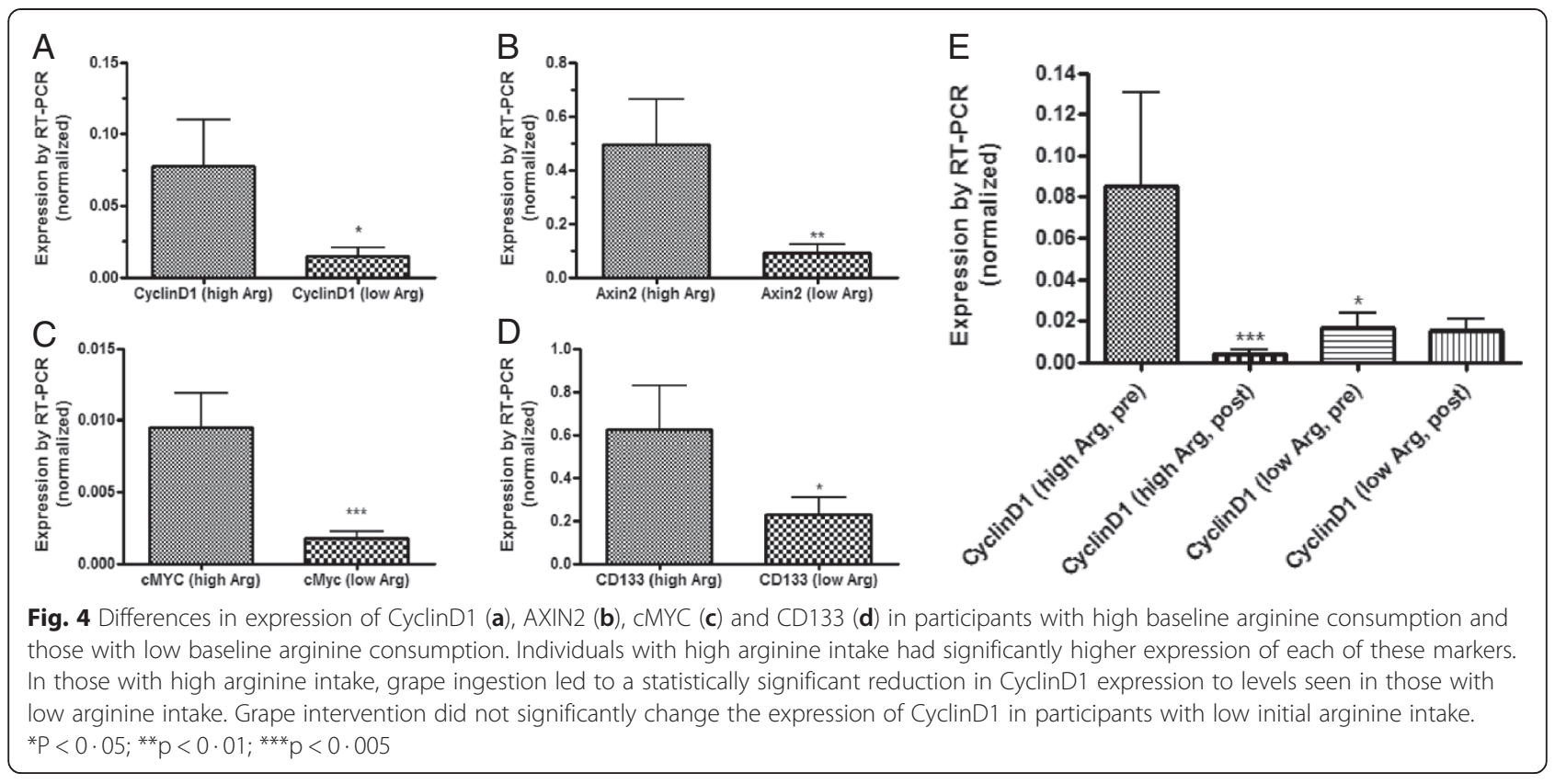

duration and did not address long-term effects on the appearance of aberrant crypt foci or pre-cancerous colonic adenomas. The significant changes noted after a short period of exposure suggests that intermittent consumption of grapes may be sufficient and this should be considered in the design of future dietary-based cancer prevention trials.

As the human colon ages, the inflammatory process leading to mucosal injury and the regenerative capacity of the epithelium are affected [19]. Telomere shortening, methylation of mucosal healing-associated genes, and alterations of growth factor signaling occur and have been postulated to affect the regenerative capacity of the epithelium. In rats, an increase in the proliferation rate of colonic mucosa is seen in conjunction with senescence [20]. Diet restriction increases intestinal apoptosis in aging rats, perhaps providing protection from an agerelated accumulation of DNA alterations [21]. Resveratrol has been shown to improve survival of mice on a high calorie diet [22], possibly through its effects on suirtuin-1 (SIRT1) activation [23]. Indeed, small molecule SIRT1 activators have been proposed for the treatment of age-related disorders [24]. In our study, colonic proliferation was significantly higher in older participants compared to younger participants. The effects of grape ingestion were most dramatically seen in the older population, with a reduction of both Wnt target genes and markers of mucosal proliferation.

High levels of dietary arginine and the polyamine synthesis pathway have been linked to colon carcinogenesis in several types of animal models [25, 26]. This has also been implicated in human colon carcinogenesis [27] and a randomized trial has demonstrated that an inhibitor of polyamine synthesis, DFMO, reduces the incidence of colon adenomas with high malignant potential [28]. Ornithine decarboxylase (ODC) catalyzes the rate-limiting step in the biosynthesis of polyamines and is inhibited by resveratrol, suggesting that one of the molecular mechanisms through which resveratrol may be operating is polyamine pathway inhibition [29]. In our study, dietary arginine consumption was ascertained through the diet survey instrument. When stratified for arginine consumption, the effect on colonic mucosal proliferation was most marked in participants who had higher levels of arginine ingestion. Based on the ongoing dietary monitoring and post-grape intervention data, this effect appears to have been due to the grape consumption and not due to a reduction in arginine consumption over the course of the study.

This study has significant implications for colon cancer prevention. The reduction in Wnt signaling and mucosal proliferation seen following relatively short-term ingestion of 1/3-1 lb of grapes should reduce the risk for the development of mutational events that can ultimately result in colon carcinogenesis. The potential benefit is most marked for older individuals and individuals whose diet is high in arginine intake. Both of these groups have an increased incidence of colon cancer [27, 30]. Subsequent studies should focus on other high-risk populations such as patients with a history of colonic adenomas (polyps) and individuals with an inherited genetic predisposition for colon cancer. Finally, patients with inflammatory bowel disease may benefit from the anti-proliferative effects of a grape-supplemented diet. 


\section{Competing interests}

The authors declare that they have no competing interests.

\section{Authors' contributions}

RFH had final oversight over all aspects of the project, was PI for the IRB/ Cancertrials.gov approvals and was the major contributor to the manuscript. MM assisted with laboratory assays and coordinated clinical trial data management and trial regulatory affairs. KP and MP directed laboratory activities and were involved in analysis of laboratory data. All authors have read and approved the final manuscript.

\section{Acknowledgements}

Supported by a grant from the Gateway for Cancer Foundation to RFH.

\section{Author details}

${ }^{1}$ Division of Hematology \& Medical Oncology, Tisch Cancer Institute, Icahn School of Medicine at Mount Sinai, One Gustav L. Levy Place, Box 1128, New York, NY 10029, USA. ²University of California, Irvine, USA.

\section{Received: 26 March 2015 Accepted: 10 June 2015}

Published online: 19 June 2015

\section{References}

1. Zanini S, Marzotto M, Fiovinazzo F, Bassi C, Bellavite P. Effects of dietary components on cancer of the digestive system. Crit Rev Food Sci and Nutr. 2014. doi:10.1080/10408398.2012.732126.

2. Terry $P$, Hu FB, Hansen $H$, Wolk A. Prospective study of major dietary patterns and colorectal cancer risk in women. Am J Epidemiol. 2001;154:1143-9.

3. Kaur M, Agarwal C, Agarwal R. Anticancer and cancer chemopreventive potential of grape seed extract and other grape-based products. J Nurt. 2009;139:1806S-12S.

4. Gullett NP, Amin ARMR, Bayraktar S, Pezzuto JM, Shin DM, Khuri FR, et al. Cancer prevention with natural compounds. Semin Oncol. 2010;37:258-81.

5. Li YA, Wu K, Huang J, Liu Y, Wang X, Meng ZJ, et al. The PTEN/PI3K/Akt and Wnt/ $\beta$-catenin pathways are involved in the inhibitory effect of resveratrol on human colon cancer cell proliferation. Int J Oncol. 2014;45:104-12.

6. Bergman M, Levin GS, Bessler H, Djaldetti M, Salman H. Resveratrol affects the cross talk between immune and colon cancer cells. Biomed Pharmacother. 2013;67:43-7.

7. Juan ME, Alfaras I, Planas JM. Colorectal cancer chemoprevention by trans-resveratrol. Pharmacological Res. 2012;65:584-91.

8. Carter LG, D'Orazio JA, Pearson KJ. Resveratrol and cancer: focus on in vivo evidence. Endocr Relat Cancer. 2014;21:R209-225.

9. Bishayee A. Cancer prevention and treatment with resveratrol: from rodent studies to clinical trials. Cancer Prev Res. 2009;2:409-18. doi:10.1158/19406207.CAPR-08-0160. Epub 2009 Apr 28.

10. Tessitore L, Davit A, Sarotto I, Caderni G. Resveratrol depresses the growth of colorectal aberrant crypt foci by affecting bax and p21CIP expression. Carcinogenesis. 2000;21:1619-22.

11. Sengottuvelan M, Viswanathan P, Nalini N. Chemopreventive effect of trans-resveratrol - a phytoalexin against colonic aberrant crypt foci and cell proliferation in 1,2-dimethylhydrazine induced colon carcinogenesis. Carcinogenesis. 2006;27:1038-46.

12. Alfaras I, Juan ME, Planas JM. Tans-resveratrol reduces precancerous colonic lesions in dimethylhydrazine-treated rats. J Agric Food Chem. 2010;58:8104-10. doi:10.1021/jf100702x.

13. Schneider Y, Duranton B, Gosse F, Schleiffer R, Seiler N, Raul F. Resveratrol inhibits intestinal tumorigenesis and modulates host-defense-related gene expression in an animal model of human familial adenomatous polyposis. Nutr Cancer. 2001;39:102-7.

14. Hope C, Planutis K, Planoutiene M, Moyer MP, Johal KS, Woo J, et al. Low concentrations of resveratrol inhibit Wnt signal throughput in colon-derived cells: Implications for colon cancer prevention. Mol Nutr Food Res. 2008;52 Suppl 1:S52-61.

15. Nguyen AV, Martinez M, Stamos MJ, Moyer MP, Planutis K, Hope C, et al. Results of a phase I pilot clinical trial examining the effect of plant-derived resveratrol and grape powder on Wnt pathway target gene expression in colonic mucosa and colon cancer. Cancer Manag Res. 2009;1:25-37.
16. Gescher AJ, Steward WP. Relationship between mechanisms, bioavailability and preclinical chemopreventive efficacy of resveratrol: A conundrum. Cancer Epidemiol Biomarkers Prev. 2003;12:953-7.

17. Kim YS, Milner JA. Bioactive food components and cancer-specific metabonomic profiles. J Biomed Biotechnol. 2011;2011:Article ID 721213, 9 pages. doi:10.1155/2011/721213.

18. Livestrong.com. 2014. http://www.livestrong.com/article/289150-grapes-forweight-loss/

19. Sipos F, Leiszter K, Tulassay Z. Effect of ageing on colonic mucosal regeneration. World J Gastroenterol. 2011;17:2981-6.

20. Holt PR, Yeh KY. Colonic proliferation is increased in senescent rats. Gastroenterology. 1988;95:1556-63.

21. Holt PR, Moss SF, Heydari AR, Richardson A. Diet restriction increases apoptosis in the gut of aging rats. J Gerontol: Biological Sci. 1998;53A:B168-72.

22. Baur JA, Pearson KJ, Price NL, Jamieson HA, Lerin C, Kalra A, et al. Resveratrol improves health and survival of mice on a high-calorie diet. Nature. 2006;16:337-42. Epub 2006 Nov 1.

23. Bauer JA, Sinclair DA. Therapeutic potential of resveratrol: the in vivo evidence. Nat Rev Drug Discov. 2006:5:493-506.

24. Hubbard BP, Sinclair DA. Small molecule SIRT1 activators for the treatment of aging and age-related diseases. Trends Pharmacol Sci. 2014;35:146-54.

25. Yerushalmi HF, Besselsen DG, Ignatenko NA, Blohm-Mangone KA, PadillaTorres $J$, Stringer DE, et al. Role of polyamines in arginine-dependent colon carcinogenesis in Apc(Min) (/+) mice. Mol Carcinog. 2006;45:764-73.

26. Gerner EW. Impact of dietary amino acids and polyamines on intestinal carcinogenesis and chemoprevention in mouse models. Biochem Soc Trans. 2007;35(Pt 2):322-5.

27. Zell J, Ignatenko NA, Yerushalmi HF, Ziogas A, Besselsen DG, Gerner EW, et al. Risk and risk reduction involving arginine intake and meat consumption in colorectal tumorigenesis and survival. Int J Cancer. 2007;120:459-68.

28. Raj KP, Zell JA, Rock CL, McLaren CE, Zoumas-Morse C, Gerner EW, et al. Role of dietary polyamines in a phase III clinical trial of difluoromethylornithine (DFMO) and sulindac for prevention of sporadic colorectal adenomas. Br J Cancer. 2013;10:512-8.

29. Wolter F, Ulrich S, Stein J. Molecular mechanisms of the chemopreventive effects of resveratrol and its analogs in colorectal cancer: key role of polyamines? J Nutr. 2004;134:3219-22.

30. Brenner H, Kloor M, Pox CP. Colorectal cancer. Lancet. 2014;383:1490-502.

\section{Submit your next manuscript to BioMed Central and take full advantage of:}

- Convenient online submission

- Thorough peer review

- No space constraints or color figure charges

- Immediate publication on acceptance

- Inclusion in PubMed, CAS, Scopus and Google Scholar

- Research which is freely available for redistribution 